\title{
Rotablation for Stent Underexpansion: An Effective Alternative
}

\author{
Michael Spartalis a, b, f, Diamantis I. Tsilimigrasc ${ }^{c}$, Demetrios Moris ${ }^{\mathrm{d}}$, \\ Christos Kontogiannis ${ }^{\mathrm{e}}$, Dimitrios C. Iliopoulos ${ }^{\mathrm{c}}$, \\ Eleftherios Spartalis ${ }^{\mathrm{c}}$
}

\begin{abstract}
A 75-year old female was evaluated for recurrent angina. She had an underexpanded stent. Conventional methods failed to achieve a full stent expansion. Rotablation resulted in an excellent angiographic outcome and full stent deployment. The patient remains angina-free at 1-year follow-up.
\end{abstract}

Keywords: Rotablation; Angiography; Stent underexpansion; Restenosis; Angioplasty

\section{Introduction}

Rotational atherectomy (RA) is a technique currently used as a different strategy for treating heavily calcified lesions during percutaneous coronary intervention (PCI), in order to facilitate optimal stent delivery and expansion [1]. RA has evolved from plaque debulking technique to plaque modification [1]. The current management of plaque modification followed by stent implantation has remarkedly reduced the incidence of acute complications, but the efficacy of this method is highly operator dependent [1].

\section{Case Report}

A 75-year old female with a history of an anterior myocardial infarction, treated with direct culprit vessel primary percuta-

Manuscript submitted December 25, 2017, accepted January 29, 2018

aDivision of Cardiology, Onassis Cardiac Surgery Center, Athens, Greece ${ }^{b}$ ESC Working Group on Thrombosis, Sophia Antipolis, France

cLaboratory of Experimental Surgery and Surgical Research, University of Athens Medical School, Athens, Greece

dDepartment of Surgery, Duke University, Durham, NC, USA

eDepartment of Clinical Therapeutics, "Alexandra" Hospital, University of Athens, Athens, Greece

${ }^{\mathrm{f} C o r r e s p o n d i n g ~ A u t h o r: ~ M i c h a e l ~ S p a r t a l i s, ~} 356$ Syggrou Ave, Athens 176 74, Greece.Email: msparta@med.uoa.gr

doi: https://doi.org/10.14740/cr670w neous coronary intervention (PCI) of left anterior descending (LAD) artery, presented with recurrent angina Canadian Cardiovascular Society class III. Stress echocardiography revealed decreased coronary flow reserve in the LAD territory. Coronary angiography showed a mid-LAD 99\% in-stent restenosis (ISR) due to stent underdeployment and an intraluminal filling defect (thrombus) (Fig. 1a). Rotablation atherectomy with 1.5 $\mathrm{mm}$ burr was attempted, and the ablation was initiated at the proximal edge of the stent. After 12 runs of rotational atherectomy using speeds of 200,000 rpm, a $3.0 \times 8 \mathrm{~mm}$ balloon was inflated to $16 \mathrm{~atm}$ within the stent, with evidence of full balloon and stent expansion, resulting in an excellent angiographic result (TIMI flow III) (Fig. 1b). At 1-year follow-up, the patient is asymptomatic.

\section{Discussion}

Drug-eluting stents (DES) have decreased the possibility of ISR. The current rate of ISR in the DES era remains higher than $10 \%$ [1]. RA in combination with DES implantation is an effective alternative approach in treating ISR [1]. The role of RA, as a treatment option for heavily calcified lesions, is well established in the literature [1-3]. Our case illustrates the clinical significance of RA in treating stent underexpansion in non-calcified lesions. Rotablation induces thermal injury and heat generation, causing the metal to erode and allowing balloon dilation and full expansion. This technique can be used when conventional methods are unsuccessful. We propose to broaden the use of rotablation in stent underexpansion, as has proven to be a safe and effective alternative [1-3].

\section{Conflict of Interest}

All authors have contributed and approved the final version of this manuscript. No author has any conflict of interest to disclose.

\section{Financial Support}

This research received no specific grant from any funding 


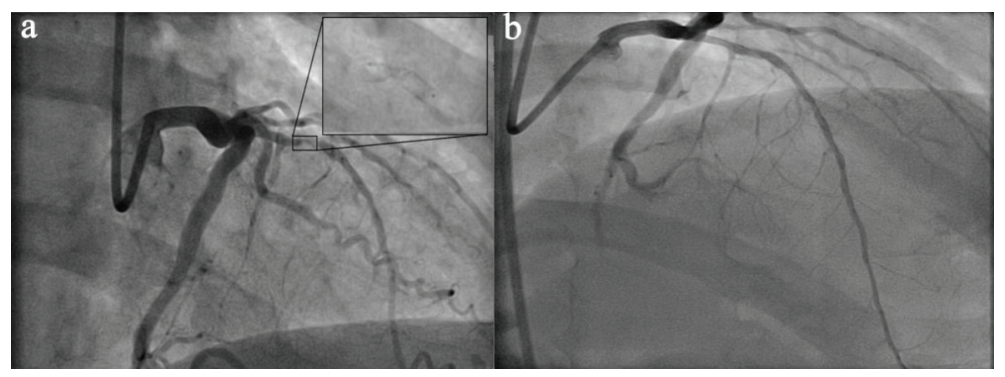

Figure 1. (a) Coronary angiography showing a mid-LAD 99\% in-stent restenosis, an angiographic image suggestive of intra-stent thrombus. (b) Coronary angiography after rotational atherectomy showing a fully expanded LAD stent with TIMI III flow.

agency, commercial or not-for-profit sectors.

\section{Consent}

Written informed consent was obtained from the patient for publication of this case report and accompanying images.

\section{References}

1. Barbato E, Shlofmitz E, Milkas A, Shlofmitz R, Azzalini L,
Colombo A. State of the art: evolving concepts in the treatment of heavily calcified and undilatable coronary stenoses - from debulking to plaque modification, a 40-year-long journey. EuroIntervention. 2017;13(6):696-705.

2. Stankovic G, Milasinovic D. Rotational atherectomy in clinical practice: the art of tightrope walking. Circ Cardiovasc Interv. 2016;9(11):e004571.

3. Sakakura K, Inohara T, Kohsaka S, Amano T, Uemura S, Ishii $\mathrm{H}$, Kadota $\mathrm{K}$, et al. Incidence and determinants of complications in rotational atherectomy: insights from the national clinical data (J-PCI Registry). Circ Cardiovasc Interv. 2016;9(11):e004278. 